\title{
Hot-Carrier Induced Degradations on RF Power Characteristics of SiGe Heterojunction Bipolar Transistors
}

\author{
Sheng-Yi Huang, Kun-Ming Chen, Member, IEEE, Guo-Wei Huang, Member, IEEE, Victor Liang, Hua-Chou Tseng, \\ Tsun-Lai Hsu, and Chun-Yen Chang, Fellow, IEEE
}

\begin{abstract}
Hot-carrier (HC) effects on high-frequency and RF power characteristics of $\mathrm{Si} / \mathrm{SiGe} \mathrm{HBT}$ are investigated in this paper. By using the two-tone load-pull measurement, we find that not only the cutoff frequency, but also the output power performances of $\mathrm{Si} / \mathrm{SiGe} \mathrm{HBT}$ are suffered by the HC stress. In this work, $S$-parameters and intrinsic elements of an equivalent hybrid- $\pi$ model were used to validate the HC effects on high-frequency characteristics. With different bias conditions, the degradations of cutoff frequency, power gain, and linearity are found to be worse under constant base-current measurement than that under constant collector-current measurement. The $\mathrm{HC}$-induced degradations on the current gain, transconductance, and ideality-factor of base and collector currents are analyzed to explain the experimental observations.
\end{abstract}

Index Terms-Cut-off frequency, hot-carrier effect, linearity, power gain, power-added efficiency, SiGe HBT.

\section{INTRODUCTION}

$\mathbf{W}$ ITH recent technology advancements that push cutoff frequency and maximum oscillation frequency over $200 \mathrm{GHz}$ [1], [2], Si/SiGe heterojunction bipolar transistors (HBTs) have become viable candidates for most microwave applications. It is known that SiGe HBTs are suited ideally for large-volume manufacturing of RF transceiver systems at and beyond $2.4 \mathrm{GHz}$, at which the silicon homojunction technologies lack performance and where SiGe HBTs provide higher integration levels than III-V component technologies. Recently, SiGe HBTs have attracted much attention for RF power applications because of their excellent microwave power performance and thermal conductivity. By optimizing the device process, the microwave power applications of SiGe-based HBT under development have moved from L-, S-, and C-band operations to X-band operation [3], [4]. Due to the high electric field at the base-emitter junction caused by the high doping levels of SiGe HBTs, the hot-carrier (HC) reliability has become a major concern for such advanced devices used in commercial products [5]. So it is worth investigating the

Manuscript received September 3, 2004; revised December 16, 2004. This work was supported in part by Semiconductor Research Corporation through Contract 2003-MJ-1089.

S.-Y. Huang and C.-Y. Chang are with the Department of Electronics Engineering and Institute of Electronics, National Chiao Tung University, Hsinchu 300, Taiwan, R.O.C.

K.-M. Chen and G.-W. Huang are with the National Nano Device Laboratories, Hsinchu 300, Taiwan, R.O.C. (e-mail: kmchen@ndl.gov.tw).

V. Liang, H.-C. Tseng, and T.-L. Hsu are with the United Microelectronics Corporation, Hsinchu 300, Taiwan, R.O.C.

Digital Object Identifier 10.1109/TDMR.2005.846829

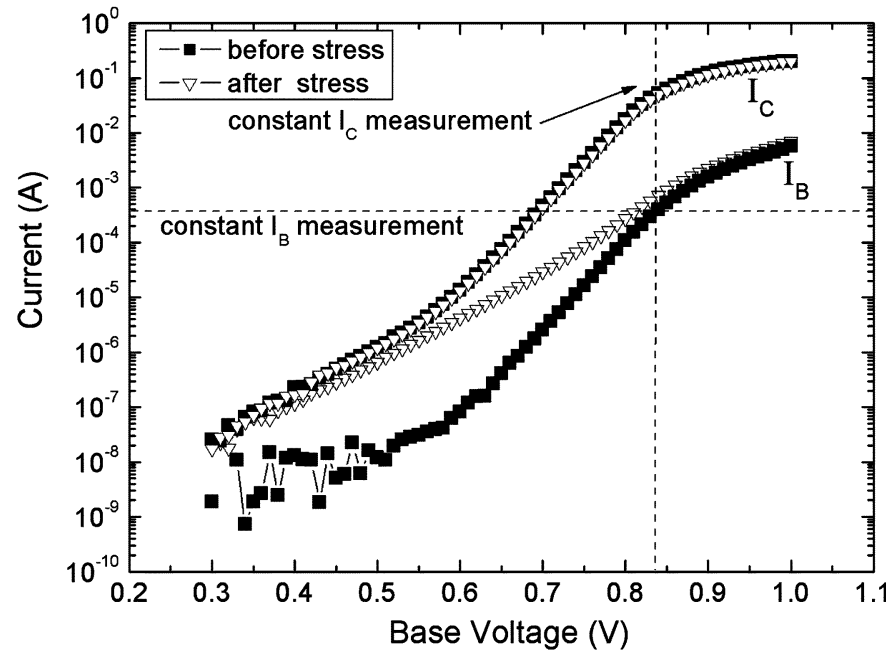

Fig. 1. Gummel plot of a typical SiGe transistor before and after stress. For power characterization, the collector current $I_{C}$ and base current $I_{B}$ are $52 \mathrm{~mA}$ and $0.34 \mathrm{~mA}$, respectively, before stress. After stress, $I_{B}$ changes to $0.7 \mathrm{~mA}$ under constant collector current measurement, while $I_{C}$ changes to $24 \mathrm{~mA}$ for constant base current measurement.

effects of hot-carrier on the high-frequency and RF power performances of SiGe HBTs. However, the most literatures on $\mathrm{HC}$ effects deal mainly with the dc characteristics and/or the low-frequency noise behavior [6]-[8], and seldom addressed the RF characteristics [9], [10].

In this paper, we investigate hot-carrier effects on the degradations of high-frequency and RF power characteristics of $\mathrm{SiGe}$ HBTs with different bias conditions. In Section II, we address the experiments of this work. The hot-carrier effects on the dc and high-frequency performances of transistors are discussed in Section III. By analyzing the intrinsic elements of an equivalent hybrid- $\pi$ model, the high-frequency behavior can be described completely. In Section IV, we present the HC effects on the RF power and linearity characteristics of devices in detail. Finally, a conclusion is given in Section V.

\section{EXPERIMENTS}

Multi-finger Si/SiGe HBTs were fabricated with a typical $0.24 \mu \mathrm{m}$ high-voltage SiGe HBT process. The nominal emitter width of the 4-finger-stripe device is $1 \mu \mathrm{m}$, and the length is $32 \mu \mathrm{m}$. Those transistors exhibit a dc current gain up to 181 and the $\mathrm{BV}_{\mathrm{CEO}}$ is $5.3 \mathrm{~V}$. The typical cutoff frequency $\left(f_{T}\right)$ and maximum oscillation frequency $\left(f_{\text {MAX }}\right)$ are about $23 \mathrm{GHz}$ and $40 \mathrm{GHz}$, respectively. The HC stress was carried out by applying 


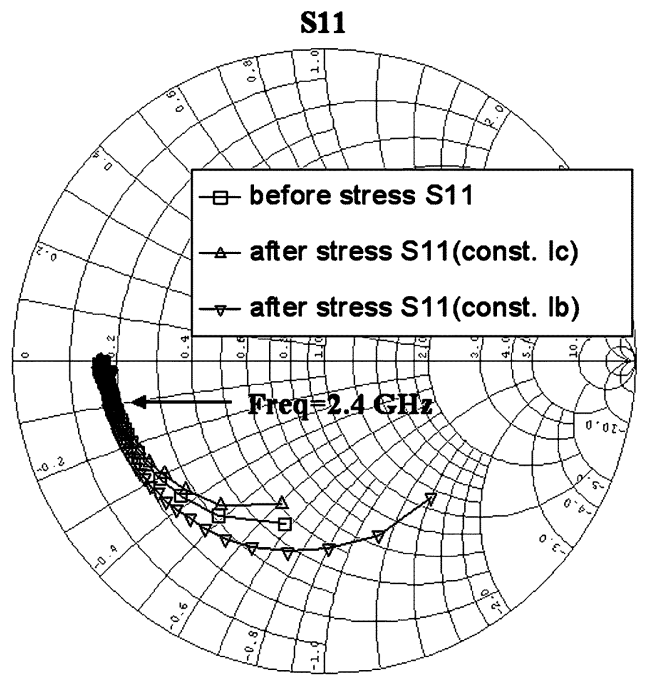

(a)

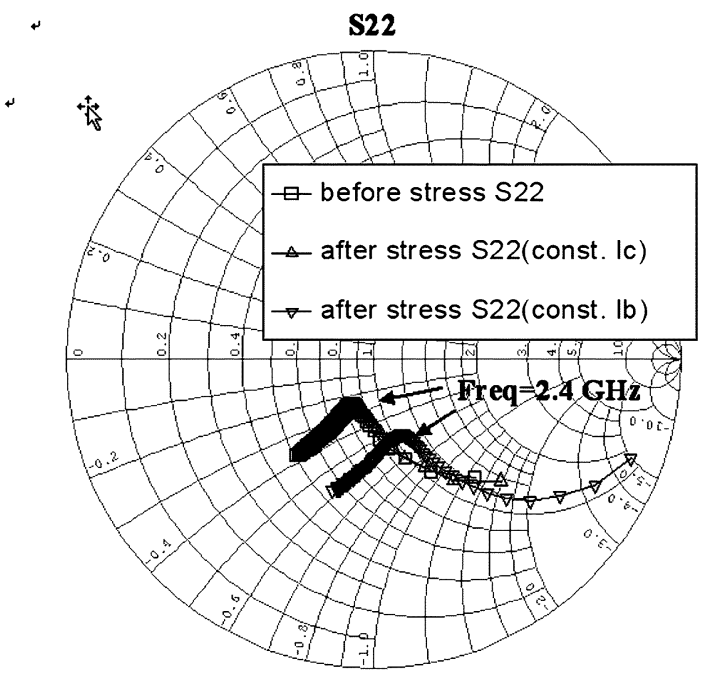

(b)

S21- polar plot

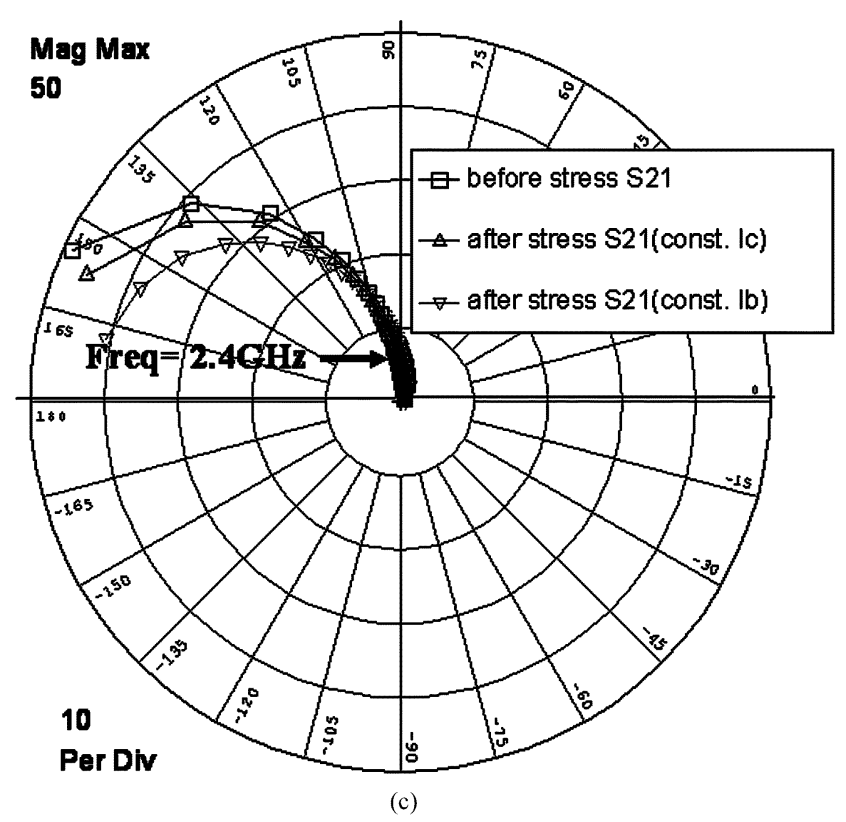

Fig. 2. Measured (a) S11, (b) S22, and (c) S21 before and after stress at constant base-current and constant collector-current measurements.

a reverse-biased base-emitter voltage $\left(V_{\mathrm{EB}}\right)$ of $3.5 \mathrm{~V}$ up to 1000 seconds at room temperature, and with the collector left open. The $S$-parameter measurements were performed by using an HP8510 network analyzer. The output power, power gain, and linearity were measured using the load-pull system, which consisted of HP85122A and ATNLP1 (power parameter extraction software), while the source and load impedances were tuned for maximum power gain and maximum output power, respectively. For the load-pull measurements, the operating frequency was chosen at $2.4 \mathrm{GHz}$, a frequency commonly used in wireless communication.

\section{HOT-CARRIER EFFECTS ON HIGH-FREQUENCY PERFORMANCE}

The Gummel plot of a typical SiGe HBT measured before and after hot-carrier stress is shown in Fig. 1. The main effect of $\mathrm{HC}$ stress on the dc characteristics is an increase of the nonideal base current and leaving the collector current unaffected, thus resulting in a degradation in the current gain of the transistor.
Hot-carrier degradation under emitter-base reverse-bias stress is one of the major reliability concerns in bipolar transistors. Reverse-biasing the emitter-base junction develops a very high electric field across the emitter-base junction, thus accelerating electrons and holes to very high velocities. These high-energy or "hot" carriers generate interface traps at the sidewall-spacer oxide and $\mathrm{Si}$ interface leading to an increase in the recombination component of the base current [11].

Since the current gain is reduced after HC stress, the high frequency and power characteristics of the transistor should be affected as well. Due to the different responses of base and collector currents under stress as shown in Fig. 1, the comparison of microwave properties under HC stress will be taken by constant basecurrent and constant collector-current measurements. For power amplifier design, either base current or collector current may be kept to a constant value when biasing the bipolar transistor.

Fig. 2 shows the HC stress effects on the $S$-parameters of a transistor with different bias measurements. The frequency 
TABLE I

Equivalent Hybrid- $\pi$ Model Elements for a Typical SiGe Transistor Before and After Stress. $V_{\mathrm{CE}}=3 \mathrm{~V}$

\begin{tabular}{|c|c|c|c|c|c|}
\hline & $\mathrm{g}_{\mathrm{m}}(\mathrm{A} / \mathrm{V})$ & $\mathrm{r}_{\pi}(\Omega)$ & $\mathrm{r}_{\text {out }}(\Omega)$ & $\mathrm{C}_{\pi}(\mathrm{pF})$ & $\mathrm{C}_{u}(\mathrm{pF})$ \\
\hline $\begin{array}{c}\text { Before stress } \\
\left(\mathrm{I}_{\mathrm{C}}=52 \mathrm{~mA}, \mathrm{I}_{\mathrm{B}}=0.34 \mathrm{~mA}\right)\end{array}$ & 2.01 & 72 & 155 & 19.2 & 0.23 \\
\hline $\begin{array}{c}\text { After stress } \\
\left(\mathrm{I}_{\mathrm{B}}=0.34 \mathrm{~mA}\right)\end{array}$ & 1.16 & 83 & 368 & 9.55 & 0.2 \\
\hline $\begin{array}{c}\text { After stress } \\
\left(\mathrm{I}_{\mathrm{C}}=52 \mathrm{~mA}\right)\end{array}$ & 1.82 & 49 & 160 & 18.96 & 0.22 \\
\hline
\end{tabular}

range is from 0.1 to $20 \mathrm{GHz}$. The $S$-parameters are the most important parameters, which are widely used for discussing the properties of microwave transistors [12]. In Fig. 2(a), we observe that $\mathrm{S} 11$ has a deviation under stress at low frequencies, it indicates the input impedance has been changed with $\mathrm{HC}$ stress. At $2.4 \mathrm{GHz}, \mathrm{S} 11$ has only a minor change as the collector current was kept constant, while S11 still has an obvious deviation as the base current was kept constant due to the reduction of the transconductance, which is resulted from the reduction of collector current. This phenomenon is different from that observation in [9], which showed the S11 has no changes under stress regardless of whether the base or the collector current was kept constant. When applying a simple equivalent hybrid- $\pi$ model in our work, we can directly extract the intrinsic element values (see Table I) from the HBT $S$-parameters after de-embedding the extrinsic parasitic. After HC stress, the dynamic base resistance $\left(r_{\pi}\right)$ referred to the SiGe HBT increases from $72 \Omega$ to $83 \Omega$ and reduces to $49 \Omega$ with the constant base-current and constant collector-current measurements respectively. The HC stress in base-emitter junction induces an increasing base current, and reduces the slope of the base current versus base-emitter voltage. Thus it increases the value of $r_{\pi}$ for constant base-current measurement. On the other hand, as to a constant collector-current measurement, the $r_{\pi}$ is reduced due to the increasing base-current. In addition, after $\mathrm{HC}$ stress, the base-emitter capacitance $\left(C_{\pi}\right)$ shows a slightly change with the constant collector-current measurement, but a large variation with the constant base-current measurement. This is resulted from the reduction of the base-emitter diffusion capacitance under a constant base-current measurement. The changes of the $r_{\pi}$ and $C_{\pi}$ under stress are consistent with the variation of $\mathrm{S} 11$.

Since $\mathrm{S} 22$ is a function of outputbias, the $\mathrm{S} 22$ of a transistor with constant base-current measurement shows a large deviation after stress due to the reduction of collector current, while that with constant collector-current measurement is changed slightly, as shown in Fig. 2(b). Furthermore, with a constant base-current measurement, the increased output resistance $\left(r_{\text {out }}\right)$ of the transistor can also validate this observation (see Table I). In Fig. 2(c), the S21, which represents the transformed gain of RF input signal, also exhibits a deviation after HC stress. Under a constant base-current measurement, the S21 deviation is larger than that under constant collector-current measurement due to the larger change of transconductance $\left(g_{\mathrm{m}}\right)$.

To validate the HC effects on the cutoff frequency $\left(f_{T}\right)$ of the transistor, we calculated the small-signal current gain $\left(h_{21}\right)$ from the $S$-parameters. Fig. 3 shows the effects of HC stress on the $h_{21}$ versus frequency. When the transistor was measured at a fixed collector current of $52 \mathrm{~mA}$, it can be seen that the magnitude of $h_{21}$ decreases after stress at low frequency regime,

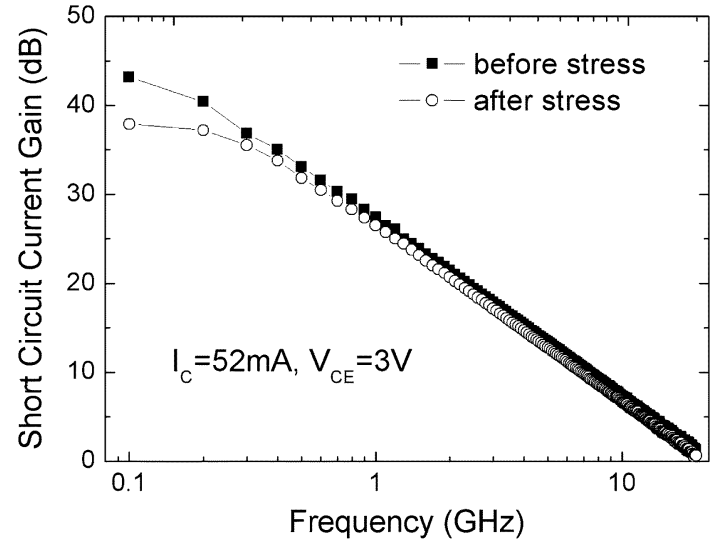

(a)

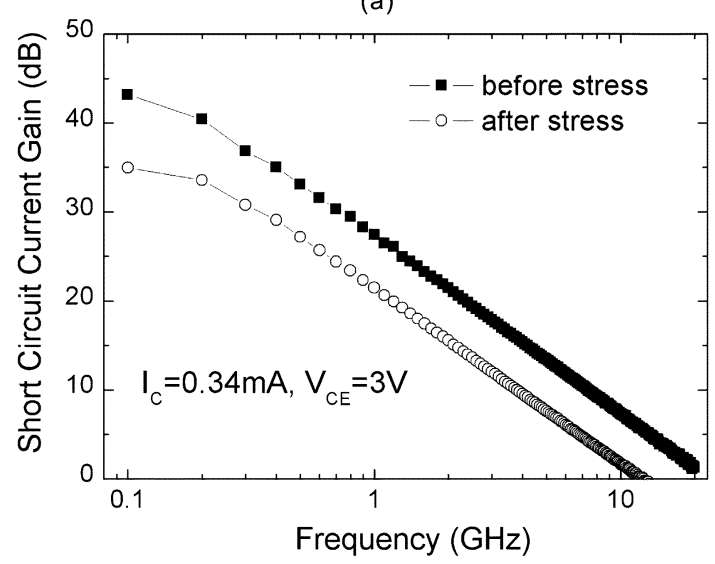

(b)

Fig. 3. The $\left|h_{21}\right|$ versus frequency before and after stress measured at (a) a fixed collector current and (b) a fixed base current.

while remains essentially unchanged for $f>1 \mathrm{GHz}$. The smallsignal current gain $h_{21}$ related to frequency $(\omega)$ in an equivalent hybrid- $\pi$ model can be expressed as [6]

$$
\left|h_{21}\right|=\frac{g_{m} r_{\pi}}{\sqrt{1+\varpi^{2} C_{\pi}^{2} r_{\pi}^{2}}} .
$$

At low frequency regime, $\left|h_{21}\right|$ approaches to the dc current gain $\left(\beta \fallingdotseq g_{m} r_{\pi}\right)$, which will decrease under stress. The reduction of $h_{21}$ at low frequency is consistent with the degradation of the dc current gain. However, at high frequency regime, $\omega^{2} C_{\pi}^{2} r_{\pi}^{2} \gg 1$, and the magnitude of $h_{21}$ approaches to $g_{m} / \omega C_{\pi}$, so it shows unchanged after $\mathrm{HC}$ stress. On the other hand, for a constant base-current measurement as shown in Fig. 3(b), $\left|h_{21}\right|$ derivation occurs after stress over the entire frequency range. This is due to the reduction of collector current after $\mathrm{HC}$ stress.

Finally, the HC effects on the cutoff frequency $\left(f_{T}\right)$ versus collector and base currents are shown in Fig. 4. It is shown that when the $f_{T}$ is measured at constant collector currents, its 


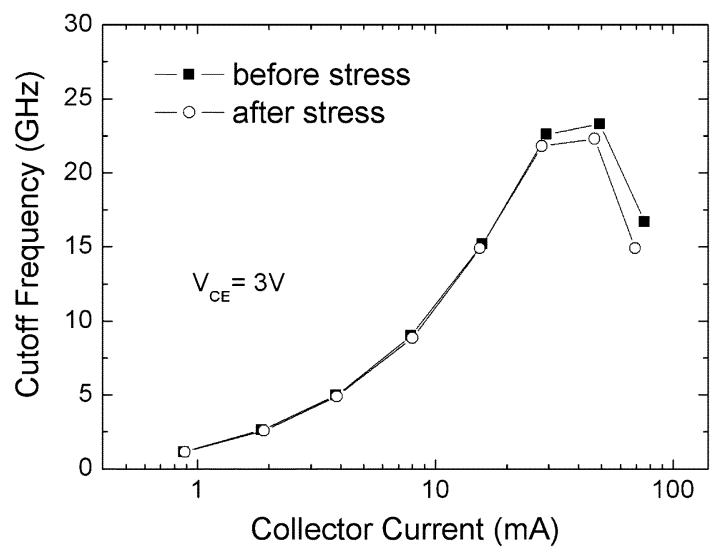

(a)

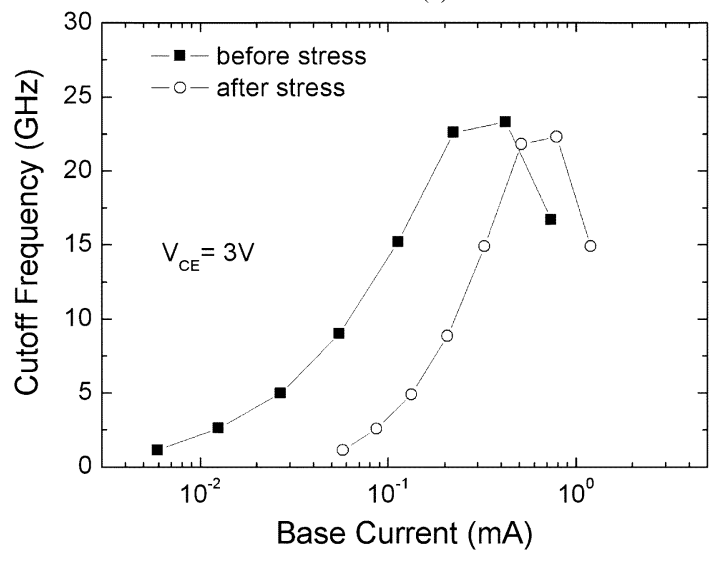

(b)

Fig. 4. (a) Cutoff-frequency versus collector current and (b) cutoff-frequency versus base current measured before and after $\mathrm{HC}$ stress.

values remain unchanged at low and medium currents, and only reduce slightly at high currents after stress (see Fig. 4(a)). On the other hand, when the $f_{T}$ is measured at constant base currents, the stress-induced degradation of $f_{T}$ is significant at low and medium currents, as shown in Fig. 4(b), due to the reduction of collector current as mentioned previously. In high current regime, where the Kirk effect might be occurred, because the device after stress has lower collector current at constant base-current measurement, it needs higher base current to enter the Kirk effect region. As a result, the measured $f_{T}$ after stress is higher than that before stress. Since the normal device operation usually would not be biased in high current region, we can conclude that the constant collector current is a better bias condition than the constant base current to reduce the HC effect on the high-frequency performance.

\section{HOT-CARRIER EFFECTS ON RF POWER BEHAVIOR}

\section{A. RF Power Characteristics}

As well as the high-frequency characteristics, the microwave power characteristics are also affected by the HC stress. Fig. 5(a) shows the effects of HC stress on the output power and power gain of a transistor measured at a fixed collector current of $52 \mathrm{~mA}$. The optimized matched source and load impedances are essentially unchanged after HC stress. After HC stress, the output power and power gain decrease slightly in the small input power regime, but show no noticeable change in the high

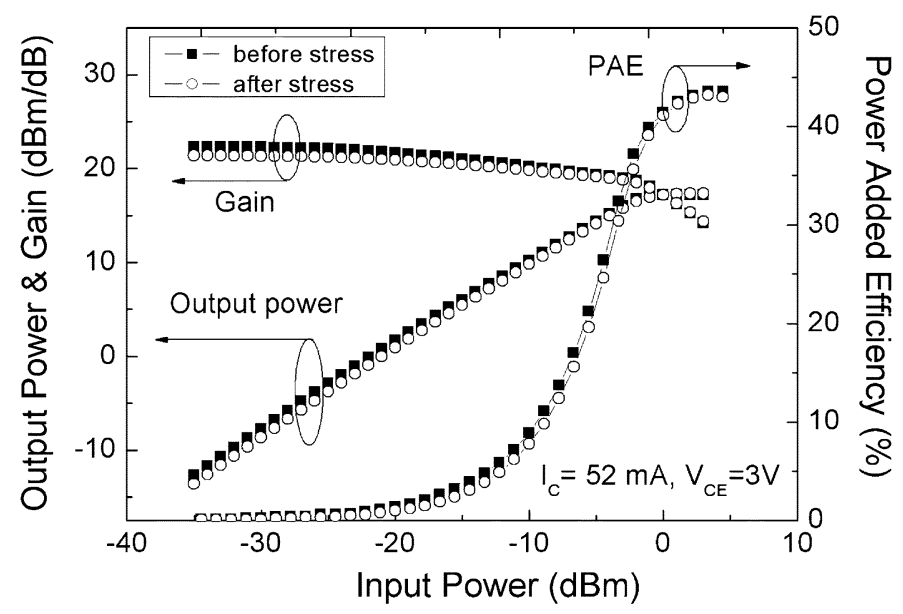

(a)

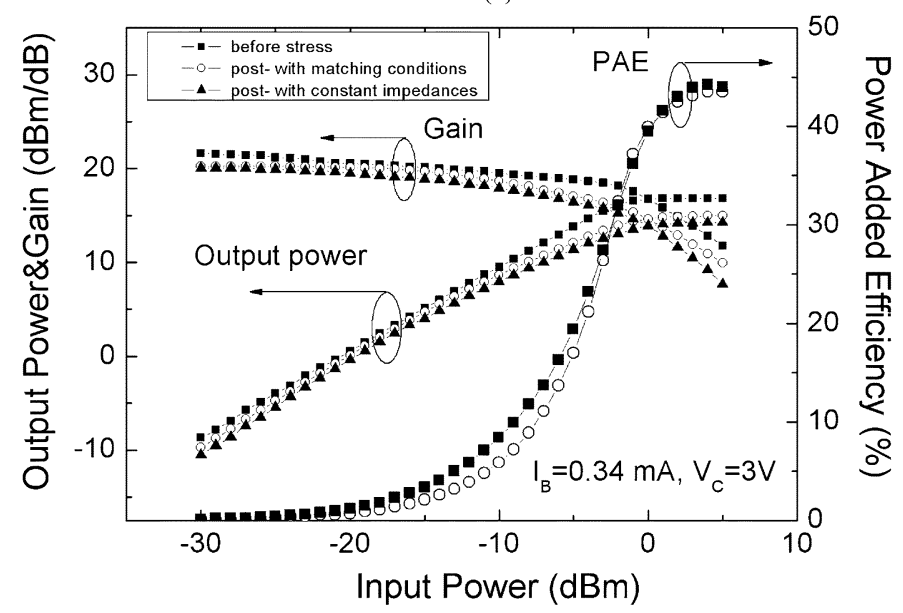

(b)

Fig. 5. (a) Linear power gain versus collector current and (b) linear power gain versus base current before and after stress measured with $P_{\text {in }}=-30 \mathrm{dBm}$.

power regime. For Class A operation, the linear power gain $G_{P}$ can be expressed as [13]

$$
G_{p}=\frac{1}{8 \pi C_{\mathrm{bc}}\left(r_{e}+r_{b}+\pi f_{T} L_{e}\right)}\left(\frac{f_{T}}{f^{2}}\right)
$$

where $r_{e}$ is the emitter resistance, $L_{e}$ is the emitter lead inductance, $C_{\mathrm{bc}}$ is the base-collector capacitance, and $f$ is the operating frequency. From (2), we know the linear power gain is proportional to $f_{T}$ approximately. Therefore, the minor reduction of linear power gain after stress is due to the minor change of $f_{T}$ as shown in Fig. 3(a). After gain compression, because the collector current is kept at a constant value, the output power and power gain even show no noticeable change under stress.

When the measurements were carried out by keeping a constant base current as shown in Fig. 5(b), the output power and power gain show significant degradations after stress. It is due to the large deviation of $f_{T}$ after stress, as shown in Fig. 3(b). It is worth noting that the optimized matched conditions have been changed after stress due to the change of the collector current. If we fixed the source and load impedances, the degradation of power performance after stress would be worse. Since the collector current is reduced after stress, it will limit the maximum magnitude of the output waveform to lower values, and thus the compression point will shift to lower output power. Hence the 


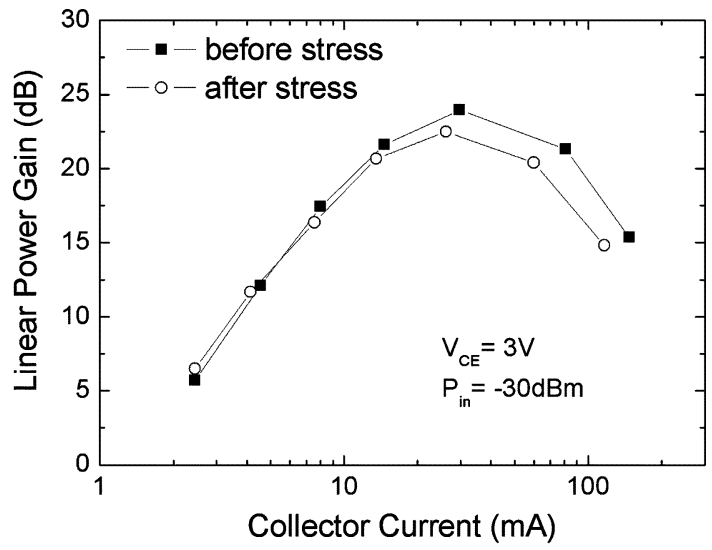

(a)

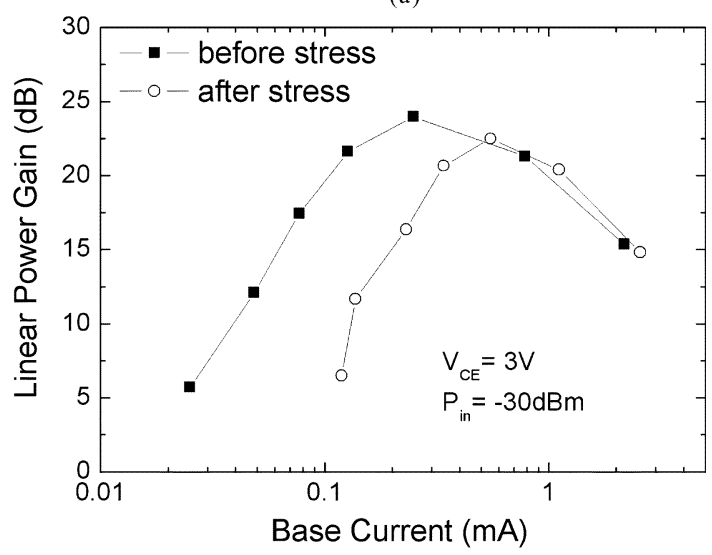

(b)

Fig. 6. Output power, power gain, and PAE versus input power before and after stress measured at (a) a fixed collector current and (b) a fixed base current.

degradation in power gain and output power will be more pronounced in the high power regime. Although the power gain is reduced after stress, the power-added efficiency (PAE) changes only slightly (from $44 \%$ to $43 \%$ ), due to the concomitant reduction of de output power dissipation.

Fig. 6 shows the linear power gain versus collector current and base current before and after stress. Comparing the measured results of Figs. 4 and 6, we know the $f_{T}$ and power gain have similar trend with $\mathrm{HC}$ stress for all bias conditions, hence the $f_{T}$ is indeed a dominant factor in (2) which affects the power gain under stress. As shown in Fig. 6(a), a little deviation of power gain can be observed under stress when the measurements were carried out by keeping a constant collector current due to the minor change of cutoff frequency. Unlike the Fig. 6(a), the effects of the HC stress on power gain will be more serious with the base driving currents, especially in the low and medium base current regimes. As shown in Fig. 6(b), the power gain degrades dramatically after stress at low and medium currents, due to the $f_{T}$ degradation. In high base current regime, the power gain shows it just slightly increases under the HC stress. From Fig. 6, it suggests that the power gain has higher immunity for hot-carrier effect when the power amplifier is biased at constant collector current.

\section{B. RF Linearity}

In practical, SiGe HBTs have a good linearity performance due to the almost complete cancellation between the output non-

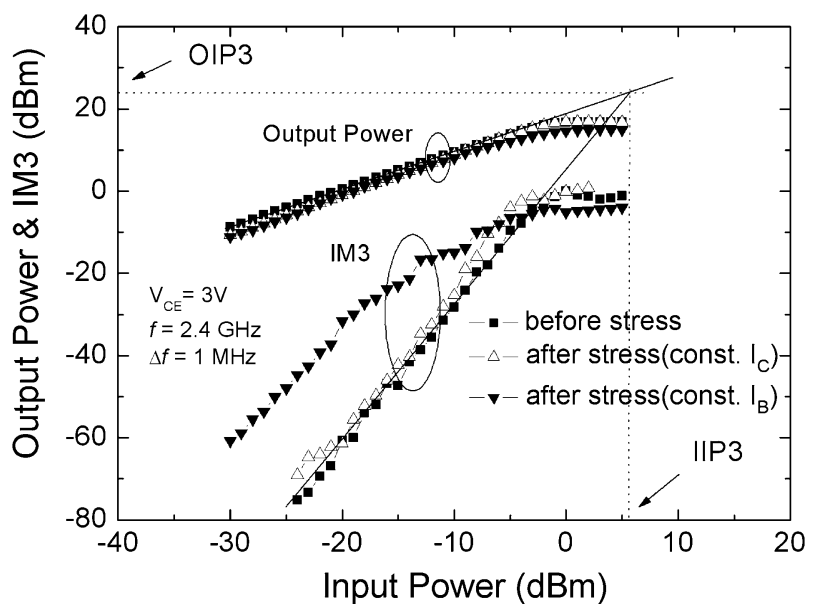

Fig. 7. Output power and third-order intermodulation power versus input power for a SiGe HBT before and after stress.

linear currents generated by the emitter-base and base-collector current sources [14]. To study the HC stress effects on the linearity of a microwave system, the two-tone load-pull measurement was also carried out [15]. As shown in Fig. 7, the thirdorder intercept point, at which the output power and third-order intermodulation (IM3) are equal, is commonly used to characterize the linearity. For low distortion operation, the third-order intercept point should be as high as possible. While the before-stress value of input third-order intercept point (IIP3) is about $6.8 \mathrm{dBm}$, the after-stress IIP3 reduces to $3.33 \mathrm{dBm}$ and $-2.46 \mathrm{dBm}$ for constant collector-current and constant basecurrent measurements, respectively.

The major nonlinear elements in a bipolar transistor are the collector current $\left(i_{C}\right)$, base current $\left(i_{b}\right)$, base-emitter charge $\left(q_{\mathrm{be}}\right)$, and base-collector charge $\left(q_{\mathrm{bc}}\right)$ [16]. Since the HC stress does not affect the base-collector junction, we only need to consider the distortion from the nonlinear exponential functions of collector current, base current, and base-emitter charge. Moreover, it is believed that in low current region, the distortion is dominated by the nonlinear contributions from transconductance [17]. As the current is increased, $g_{m}$ nonlinearities are decreased due to the increase of $g_{m}$ and the feedback effect of emitter and base resistances. When measured at the same collector current, the transconductance is changed slightly and it still has a high value before and after stress. Nevertheless, the ideality factor of base current $\left(n_{b}\right)$ has changed drastically from 1.005 to 1.52 after stress, while the ideality factor of collector current is still about 1 . It indicates that partial IM3 cancellation, which has been attributed to the interaction of $i_{b}$ and $i_{c}$, or $i_{c}$ and $q_{\text {be }}$, has been reduced [16]. So the reduction of IIP3 after stress is mainly due to the reduced cancellation effect. As to the constant base-current measurement, not only $n_{b}$ increases to 1.67 , but the related collector current also reduces to a lower value after HC stress. Consequently, the degradation of linearity measured at constant base current is much worse than that measured at constant collector current.

\section{CONCLUSION}

In this paper, we have investigated in detail the hot-carrier effects on the high-frequency and RF power characteristics of 
$\mathrm{Si} / \mathrm{SiGe} \mathrm{HBTs}$. Due to the increase of nonideal base current after stress, the current gain will be decreased. Consequently, we find that the cutoff frequency, output power, power gain, and linearity are suffered by the HC stress. By comparing stress effects at various bias conditions, we observe that the performance degradation is much smaller under constant collector-current measurement, compared to constant base-current measurement. Because of the increased ideality factor of the base current and the reduced collector current after stress, the high-frequency and power characteristics show larger stress-induced degradations for constant base-current measurements. It therefore suggests that the $\mathrm{Si} / \mathrm{SiGe}$ amplifier performance can be made more robust to $\mathrm{HC}$ effects by biasing the HBT at constant collector current.

\section{ACKNOWLEDGMENT}

The authors would like to thank the staff of United Microelectronics Corporation for their helpful comments.

\section{REFERENCES}

[1] J.-R. Rieh, B. Jagannathan, H. Chen, K. T. Schonenberg, D. Angell, A. Chinthakindi, J. Florkey, F. Golan, D. Greengerg, S.-J. Jeng, M. Khater, F. Pagette, C. Schnabel, P. Smith, A. Stricker, K. Vaed, R. Volant, D. Ahlgren, G. Freenman, K. Stein, and S. Subbanna, "SiGe HBTs with cutoff frequency of $350 \mathrm{GHz}$," in IEDM Tech. Dig., 2002, pp. 771-774.

[2] B. Jagannathan, M. Khater, F. Pagette, J.-S. Rieh, D. Angell, H. Chen, J. Florkey, F. Golan, D. R. Greenberg, R. Groves, S. J. Jeng, J. Johnson, E. Mengistu, K. T. Schonenberg, C. M. Schnabel, P. Smith, A. Stricker, D. Ahlgren, G. Freeman, K. Stein, and S. Subbanna, "Self-aligned SiGe NPN transistors with $285 \mathrm{GHz} \mathrm{f}_{\mathrm{MAX}}$ and $207 \mathrm{GHz} \mathrm{f}_{T}$ in a manufacturable technology," IEEE Electron Device Lett., vol. 23, no. 5, pp. 258-260, May 2002.

[3] J. N. Burghartz, J.-O. Plouchart, K. A. Jenkins, C. S. Webster, and M. Soyuer, "SiGe power HBTs for low-voltage, high performance RF applications," IEEE Electron Device Lett., vol. 19, no. 4, pp. 103-105, Apr. 1998.

[4] Z. Ma, S. Mohammadi, P. Bhattacharya, L. P. B. Katehi, S. A. Alterovitz, and G. E. Ponchak, "A high-power and high-gain X-band Si/SiGe/Si heterojunction bipolar transistor," IEEE Trans. Microwave Theory Tech., vol. 50, no. 4, pp. 1101-1108, Apr. 2002.

[5] L. Vendrame, P. Pavan, G. Corva, A. Nardi, A. Neviani, and E. Zanoni, "Degradation mechanism in polysilicon emitter bipolar junction transistors for digital application," Microelectron. Rel., vol. 40, pp. 207-230, 2000.

[6] A. Neugroschel, C. T. Sah, and M. S. Carroll, "Degradation of bipolar transistor current gain by hot holes during reverse emitter-base bias stress," IEEE Trans. Electron Devices, vol. 43, no. 8, pp. 1286-1290, Aug. 1996.

[7] U. Gogineni, J. D. Cressler, G. Niu, and D. L. Harame, "Hot electron and hot hole degradation of UHV/CVD SiGe HBTs," IEEE Trans. Electron Devices, vol. 47, no. 7, pp. 1440-1448, Jul. 2000.

[8] P. Llinares, S. Niel, G. Ghibaudo, L. Vendrame, and J. A. Chroboczek, "Retarding effect of surface base compensation on degradation of noise characteristics of BICMOS BJTs," Microelectron. Rel., vol. 37, pp. 1603-1606, 1997.

[9] M. Borgarino, J. G. Tartarin, J. Kuchenbecker, T. Parra, H. Lafontaine, T. Kovacic, R. Plana, and J. Graffeuil, "On the effects of hot carriers on the RF characteristics of $\mathrm{Si} / \mathrm{SiGe}$ heterojunction bipolar transistor," IEEE Microw. Guided Wave Lett., vol. 10, no. 11, pp. 466-468, Nov. 2000.

[10] J. Kuchenbecker, M. Borgarino, L. Bary, G. Cibiel, O. Llopis, J. G. Tartarin, J. Graffeuil, S. Kovacic, J. L. Roux, and R. Plana, "Reliability investigation in SiGe HBTs," in Proc. Silicon Monolithic Integrated Circuits in RF Systems, 2001, pp. 131-134.
[11] S. A. Peterson and G. P. Li, "Hot carrier effects in advanced self-aligned bipolar transistors," in IEDM Tech. Dig., 1985, pp. 22-25.

[12] F. Schwierz and J. J. Liou, Modern Microwave Transistors. New York: Wiley, 2002.

[13] G. B. Gao, H. Morkoc, and M. C. Frank, "Heterojunction bipolar transistor design for power applications," IEEE Trans. Electron Devices, vol. 39, no. 9, pp. 1987-1997, Sep. 1992.

[14] W. Kim, S. Kang, K. Lee, M. Chung, J. Kang, and B. Kim, "Analysis of nonlinear behavior of power HBTs," IEEE Trans. Microwave Theory Tech., vol. 50, no. 7, pp. 1714-1722, Jul. 2002.

[15] Y. Yang, J. Yi, J. Nam, B. Kim, and M. Park, "Measurement of twotone transfer characteristics of high-power amplifiers," IEEE Trans. Microwave Theory Tech., vol. 49, no. 3, pp. 568-571, Mar. 2001.

[16] M. Iwamoto, P. M. Asbeck, T. S. Low, C. P. Hutchinson, J. B. Scott, A. Cognata, Q. Xiaohui, L. H. Camnitz, and D. C. D'Avanzo, "Linearity characteristics of GaAs HBTs and the influence of collector design," IEEE Trans. Microwave Theory Tech., vol. 48, no. 12, pp. 2377-2388, Dec. 2000.

[17] G. Niu, Q. Liang, J. D. Cressler, C. S. Webster, and D. L. Harame, "RF linearity characteristics of SiGe HBTs," IEEE Trans. Microwave Theory Tech., vol. 49, no. 9, pp. 1558-1565, Sep. 2001.

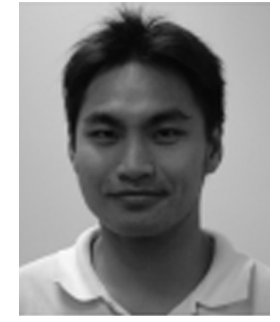

reliability.
Sheng-Yi Huang was born in Yunlin, Taiwan, R.O.C., in 1978. He received the B.S. degree in electrical engineering from National Cheng Kung University, Tainan, Taiwan, in 2001, and the M.S.E.E. degree from the National Chiao Tung University (NCTU), Hsinchu, Taiwan, in 2003. He is currently working toward the Ph.D. degree in electronic engineering at NCTU.

His current research focuses on mixed-mode and RF CMOS/BiCMOS device issues including device modeling, noise characteristics, power behavior, and

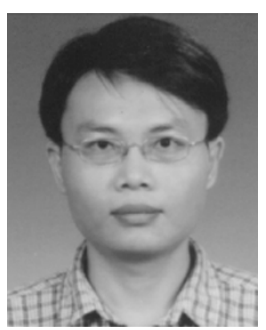

Kun-Ming Chen (M'01) received the M.S. and $\mathrm{Ph} . \mathrm{D}$. degrees in electronics engineering from National Chiao Tung University, Hsinchu, Taiwan, R.O.C., in 1996 and 2000, respectively.

He joined the National Nano Device Laboratories, Hsinchu, Taiwan, in 2000 as an Associate Researcher. $\mathrm{He}$ has been engaged in research on microwave device process and characterization.

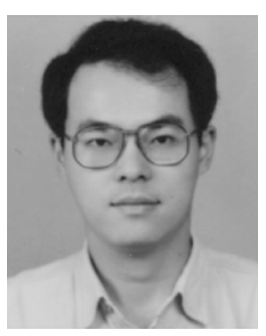

Guo-Wei Huang (S'94-M'97) was born in Taipei, Taiwan, R.O.C., in 1969. He received the B.S. degree in electronics engineering and the Ph.D. degree from National Chiao Tung University, Taiwan, in 1991 and 1997, respectively.

He joined National Nano Device Laboratories, Hsinchu, Taiwan, in 1997 as an Associate Researcher. His current research interests focus on microwave device design, characterization, and modeling.
Victor Liang, photograph and biography not available at the time of publication. 


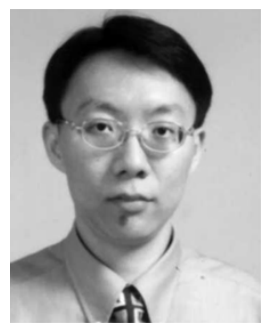

Hua-Chou Tseng received the B.S. degree and the $\mathrm{Ph} . \mathrm{D}$. degree in electronic engineering from National Chiao Tung University (NCTU), Taiwan, in 1991 and 1997, respectively. In his Ph.D. program, his research interests included low temperature $\mathrm{Si} / \mathrm{SiGe}$ selective epitaxial growth and gas source doping for ultra-shallow junction formation.

After graduating from NCTU, he joined United Microelectronics Corporation (UMC) to develop the low-power 6T-SRAM, including the process integration and the device tuning of $0.25 \mu \mathrm{m}, 0.18$ $\mu \mathrm{m}$, and $0.15 \mu \mathrm{m}$ generations, from 1997 to 2000 . He is currently the group leader of RF-CMOS and SiGe-BiCMOS technology development at UMC.

Dr. Tseng is the international technical committee member of the International SiGe Technology and Device Meeting (ISTDM).

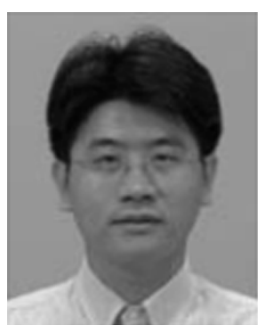

Tsun-Lai Hsu was born in Chi-I, Taiwan, R.O.C., in 1968. He received the B.S. degree in electric engineering from National Ocean University in 1990 and the Master and Ph.D. degrees in electrical engineering from National Tsing Hua University (NTHU), Taiwan, in 1992 and 1996, respectively. In his Ph.D. program, his research interests included reliability study, base resistance physical analysis, 1/f noise measurement for poly-emitter BJT.

After graduation from NTHU, he joined ERSO/ITRI to develop the RF double-poly BJT technology, including RF/Noise characteristics measurement and analysis. He worked on $0.18 \mu \mathrm{m}$ SiGe-BiCMOS technology development at United Microelectronics Corporation (UMC) in 2001 and 2002 and he has been working in RF passive/active devices design for RF-CMOS at United Microelectronics Corporation (UMC) since 2002. His current researches are focused on inductor, substrate noise isolation, and RF de-embedding methodology designs.

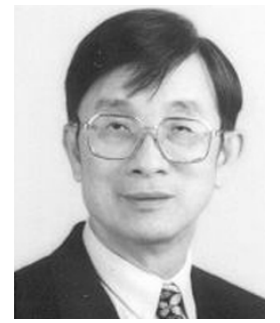

Chun-Yen Chang (F'88) was born in Feng-Shan, Taiwan, R.O.C. He received the B.S. degree in electrical engineering from Cheng Kung University, Taiwan, in 1960, and the M.S. degree in tunneling in semiconductor-supercondutor junctions and the $\mathrm{Ph} . \mathrm{D}$. degree in carrier transport across metal-semiconductor barrier, both from National Chiao-Tung University (NCTU), Hsinchu, Taiwan, in 1969.

$\mathrm{He}$ has devoted himself to education and academic research for more than 40 years. He has contributed profoundly to the areas of microelectronics and optoelectronics, including the invention of the method of low-pressure-MOCVD-using tri-ethyl-gallium to fabricate LED, laser, and microwave transistors, $\mathrm{Zn}$-incorporation of $\mathrm{SiO}$ for stabilization of power devices, and nitridation of $\mathrm{SiO}$ for ULSIs, etc. From 1962 to 1963, he fulfilled his military service by establishing at NCTU Taiwan's first experiment TV transmitter that formed the founding structure of today's CTS. In 1963, he joined NCTU to serve as an instructor establishing a high vacuum laboratory. In 1964, he and his colleague established the semiconductor research center (SRC) at NCTU with a very up-to-date, albeit homemade, facility for silicon device processing, where they made the nation's first Si Planar transistor in April 1965, and subsequently the first IC in August 1966. In 1968, he published Taiwan's first-ever semiconductor paper in the international journal Solid State Electronics. In 1969, he became a Full Professor, teaching solid state physics, quantum mechanics, semiconductor devices, and technologies. From 1977 through 1987, he single-handedly established a strong electrical engineering and computer science program at NCKU where GaAs, $\alpha-\mathrm{Si}$, poly-Si researches were established in Taiwan for the first time. Since 1987 he served consecutively as Dean of Research (1987-1990), Dean of Engineering (1990-1994), and Dean of Electrical Engineering and Computer Science (1994-1995). Simultaneously, he was serving as the founding president of National Nano Device Laboratories (NDL) from 1990 through 1997. In 1997, he became Director of the Microelectronics and Information System Research Center (MIRC), NCTU (1997-1998). Many of his former students have since become founders of the most influential Hi-Tech enterprises in Taiwan, namely UMC, TSMC, Winbond, MOSEL, Acer, Leo, etc. On August 1, 1998, he was appointed as the President of NCTU. As the National-Chair-Professor and President of NCTU, his vision is to lead the university for excellence in engineering, humanity, art, science, management, and bio-technology. To strive forward to world class multidisciplinary university is the main goal to which he and his colleagues have committed.

Dr. Chang received the IEEE Third Millennium Medal in 2000. He is a member of Academia Sinica and a Foreign Associate of the National Academy of Engineering. 